\title{
O Momento do Diagnóstico e as Dificuldades Encontradas pelos Oncologistas Pediátricos no Tratamento do Câncer em Belo Horizonte
}

\author{
The Moment of Diagnosis and the Difficulties Encountered by Pediatric \\ Oncologists in the Treatment of Cancer in Belo Horizonte
}

Júlia Dias Santana Malta', Virgínia Torre Schall², Celina Maria Modena ${ }^{3}$

\section{Resumo}

A percepção e reação da criança diante da doença são influenciadas pela atitude dos pais que, por sua vez, é determinada pela postura do médico frente à patologia e ao tratamento. $\mathrm{O}$ objetivo deste artigo foi descrever o momento da comunicação do diagnóstico do câncer infantil para o paciente e a família, e as principais dificuldades enfrentadas pelos oncologistas pediátricos no tratamento da criança com câncer. Como critério de escolha de sujeitos para as entrevistas, os oncologistas precisavam ter especialização e/ ou residência em oncologia pediátrica. Foram entrevistados cinco oncologistas pediátricos. As entrevistas semiestruturadas foram gravadas com a autorização dos entrevistados e o material foi analisado através da análise temática. Essa análise permitiu identificar três grandes categorias em relação ao momento de confirmar o diagnóstico de câncer infantil e as dificuldades encontradas no tratamento da doença: 1) A verdade deve ser dita para as crianças e cuidadores; 2) As dificuldades encontradas na organização e estrutura do serviço de oncologia; 3) A questão da humanização. A comunicação do diagnóstico é um momento delicado para o paciente, familiares e médicos. O oncologista, pelo seu papel de detentor de conhecimento, deve oferecer aos pais a compreensão necessária sobre a doença para que estes possam suportar a doença e o tratamento do filho sem gerar sofrimento neles próprios. Em relação às dificuldades encontradas pelos médicos no tratamento do câncer, o Sistema Único de Saúde (SUS) precisa ser cúmplice na promoção da saúde, prevenção e tratamento de doenças.

Palavras-chave: Oncologia; Diagnóstico; Pediatria; Efeitos psicossociais da doença; Cuidado da criança; Neoplasias

\footnotetext{
${ }^{1}$ Mestrado em Saúde Coletiva no Instituto de Pesquisa René Rachou - FIOCRUZ

${ }^{2}$ Doutorado em Educação pela Pontifícia Universidade Católica do Rio de Janeiro - PUCRJ

${ }^{3}$ Pós-Doutorado em Saúde Coletiva pela FIOCRUZ

Autores filiados ao Instituto de Pesquisa René Rachou - FIOCRUZ, Laboratório de Educação em Saúde

Endereço para correspondência: Júlia Dias Santana Malta. Av. Afonso XIII, 820/301 - Gutierrez - Belo Horizonte (MG), Brasil - CEP: 30430-170.

E-mail: jdsfisio@yahoo.com.br
} 


\section{INTRODUÇÃO}

"Cada palavra dita por um médico ao seu paciente é um veredicto. Assim como o escritor, ele deve avaliar cada palavra e saber usá-la com extremo rigor."

Moacyr Scliar, médico sanitarista e escritor

O diagnóstico do câncer pediátrico desdobra-se em dois momentos para as mães: o do alívio em saber o que seu filho tem, e o temor misturado à sensação de que o médico sela o seu destino e o de seu filho com a sua palavra. Em muitos casos, percebe-se que receber o diagnóstico de câncer é como receber uma sentença de morte devido a todo o peso que esta palavra carrega.

Já a reação da criança diante da doença está diretamente relacionada a múltiplos fatores, tais como: idade, estresse imediato representado pela dor física desencadeada pela doença, traços de personalidade, experiências e qualidade de suas relações parentais. Em certos casos, ela será separada da família para entrar no contexto de uma instituição que passará a fazer parte de sua vida. Aparecerão personagens novos com os quais ela passará a estabelecer relações bastante duradouras: os profissionais de saúde ${ }^{1}$.

Pacientes e familiares esperam que o médico seja amistoso, cordial, gentil, carinhoso e solidário no seu sofrimento, oferecendo a eles o apoio emocional de que carecem. Expressam o desejo de serem acolhidos, de forma cuidadosa, e personalizados, porém o médico não é ativamente estimulado a pensar no paciente em sua inteireza, como um ser biopsicossocial, e a perceber o significado do adoecer para o paciente e seus familiares ${ }^{2}$.

A essência da prática clínica com crianças é diferente de qualquer dos campos da medicina clínica. São, no mínimo, duas as perspectivas a considerar: uma diz respeito à doença da criança, com todos os componentes de sofrimento físico e emocional, e a outra é o impacto desse sofrimento sobre a criança e a família. Para atender com qualidade um paciente da oncologia pediátrica, o médico deve ser possuidor de um conjunto amplo de competências e de habilidades específicas na comunicação com as crianças, seus pais ou cuidadores diretos e com outros membros da família ${ }^{3}$.

A comunicação com os profissionais de saúde foi uma queixa de vários cuidadores de crianças com câncer em um trabalho que visava a discutir o amparo médico. A maioria não se sentia acolhida e relatou não receber atenção e informações suficientes e claras daqueles que estavam cuidando de suas crianças ${ }^{4}$.

Muitos cuidadores reclamaram a respeito da falta de diálogo existente entre eles e os médicos, porém, aparentemente, esse diálogo existe, mas não alcança a compreensão desses familiares. Profissionais de saúde e pacientes veem os problemas de saúde de maneiras muito diferentes, já que suas perspectivas estão baseadas em premissas distintas. Para os pacientes e cuidadores, o adoecer envolve as experiências subjetivas de mudanças físicas ou emocionais e a confirmação dessas mudanças por parte de outras pessoas. Para o profissional, um problema de saúde é fundamentado nas mudanças físicas de estrutura e de funcionamento do organismo que podem ser demonstradas objetivamente e quantificadas com base nas mensuraçōes fisiológicas normais. Trata-se de uma relação assimétrica em que o médico detém um corpo de conhecimentos do qual o paciente geralmente é excluído5. Para um bom diálogo entre médico e paciente/cuidador, é necessário que barreiras como estas sejam transpostas para que os médicos consigam chegar até seus pacientes. Depois de realizar um trabalho envolvendo a percepção das crianças com câncer e seus cuidadores, o objetivo deste artigo foi descrever o momento da comunicação do diagnóstico do câncer infantil para o paciente e a família e as principais dificuldades enfrentadas pelos oncologistas pediátricos no tratamento da criança com câncer.

\section{METODOLOGIA}

O presente trabalho foi embasado na abordagem qualitativa que incorpora a questão do significado como inerente aos atos e às relaçôes considerando o sujeito do estudo com suas crenças, valores e significados. Sua análise não se preocupa em quantificar, mas sim em compreender e explicar a dinâmica das relaçôes sociais ${ }^{6}$.

Como critério de escolha de sujeitos para as entrevistas, os oncologistas precisavam ter especialização e/ ou residência em oncologia pediátrica. Foram entrevistados cinco oncologistas pediátricos que correspondem a todos os especialistas no tratamento do câncer infantil de Belo Horizonte. Esses profissionais estavam atendendo em hospitais e centros especializados para o tratamento do câncer no período de junho a agosto de 2007. No Brasil, poucas são as instituições que oferecem residência e especialização em oncologia pediátrica e no Sudeste esta oferta acontece em São Paulo. Portanto, atualmente, existem apenas cinco médicos com este título atuando em Belo Horizonte, três mulheres e dois homens com idade entre 30 e 37 anos. As entrevistas foram realizadas em seus locais de trabalho.

As entrevistas semiestruturadas foram gravadas com a autorização dos entrevistados e, posteriormente, transcritas. Para essa coleta de informações, foram 
formuladas questôes norteadoras a respeito do momento do diagnóstico e das dificuldades encontradas nesta área de atuação da medicina que serviram de fios condutores segundo a metodologia 7 .

Para a análise do material obtido nas entrevistas, foi utilizado o procedimento da análise temática, que consiste em "descobrir os núcleos de sentido que compõem uma comunicação cuja presença ou frequência signifiquem alguma coisa para o objetivo analítico visado"6.

Todos os aspectos do estudo foram aprovados pelo Comitê de Ética do Instituto de Pesquisa René Rachou, parecer no 17/2006 - CEPSH-CPqRR. Os cuidadores assinaram o termo de consentimento e a identidade de todos os participantes permanecerá em sigilo.

\section{RESULTADOS E DISCUSSÕES}

A análise temática das entrevistas permitiu identificar três grandes categorias em relação ao momento de confirmar o diagnóstico de câncer infantil e as dificuldades encontradas no tratamento da doença: 1) A verdade deve ser dita para as crianças e cuidadores; 2) As dificuldades encontradas na organização e estrutura do serviço de oncologia; 3) A questão da humanização.

\section{A VERDADE DEVE SER DITA PARA AS CRIANÇAS E CUIDADORES}

A comunicação no cuidado médico abrange os conceitos de confidencialidade, relato da verdade, consentimento esclarecido e comportamento ético. A informação ou relato da verdade é um dos conceitos médicos mais antigos, sendo que, em algumas culturas da antiguidade, a função do médico era, muitas vezes, mais informativa do que curativa ${ }^{8}$.

O relato da verdade tem mostrado ser um importante instrumento terapêutico. A informação diminui o sentimento de isolamento do paciente e colabora para uma cooperação mútua na relação médico-paciente. Saber o nome e entender as implicaçóes de sua doença possibilita ao paciente repensar-se frente a esta realidade, programar sua vida dentro de novos contornos e atuar a partir de alternativas reais com a autonomia e controle possível a que tem direito?.

Em Pediatria, a questão da transmissão do diagnóstico adquire outras nuances por ser a criança um sujeito tutelado pelos pais ou responsáveis, deles dependente, com autonomia, na melhor das hipóteses, relativa. Antigamente, os pais, responsáveis e médicos pensavam saber o que era melhor para a criança e atuavam com a intenção de protegê-la. A transmissão do diagnóstico a ela (criança) raramente era encarada como uma tarefa.
A partir da década de 1970, tornou-se mais comum a comunicação do diagnóstico à criança, principalmente entre profissionais americanos e europeus ${ }^{10}$. Esta nova perspectiva de trabalho iniciou-se, sobretudo, na área de oncologia. Até então, pensava-se com frequência na criança como um sujeito incapaz de ter entendimento da doença, morte, sofrimento, tratamentos e que deveria ser "protegida" do impacto emocional determinado por essas duras realidades (como se fosse possível tratar a criança doente apesar dela mesma).

A nova visão de infância que vem se estabelecendo, principalmente a partir da influência da psicanálise na Pediatria, do reconhecimento dos efeitos benéficos de manter a criança a par de seu diagnóstico e tratamento, e uma crescente movimentação de profissionais comprometidos com a dimensão ética das relações médico-paciente determinaram novas posturas frente à revelação do diagnóstico à criança ${ }^{11}$.

Os cinco oncologistas pediátricos entrevistados concordaram sobre a importância de se dizer toda a verdade para as crianças.

Primeiro eu converso com os pais [...], mas depois a gente tem que conversar com a criança na linguagem dela. Tem que ser feito e não adianta a criança ficar iludida. Ela é a maior parceira no tratamento. Uma criança que desconhece o que está sendo feito com ela vai se sentir insegura, além de achar que estão mentindo pra ela. Isso interfere muito na condução do tratamento [...]. Se não sabem, viram objetos do tratamento e não sujeitos. OP2

A criança tem que saber a verdade porque senão ela não vai ajudar a gente no tratamento. A criança que sabe, que escuta vai levar o tratamento de uma forma melhor. Ela vai confiar mais em você. Senão, a criança começa a esconder o que está sentindo porque fica com medo de contar. OP1

A comunicação da verdade sobre uma doença é um processo no qual se pode distinguir os seguintes aspectos básicos: preparação pessoal e lugar físico, compartilhar a informação de acordo com o entendimento do sujeito, exploração do desejo de receber informações, acolher os sentimentos do sujeito ${ }^{12}$.

\section{I.a) Preparação pessoal e lugar físico:}

Inclui a disposição de tempo e calma. É importante estar em um lugar privado onde o médico, o paciente e o acompanhante possam ficar tranquilos e onde o paciente possa processar a recente informação junto aos seus familiares. 
Se o paciente está internado, a gente tira essa criança com a família do leito e leva para uma salinha pra tudo ficar mais tranquilo, pra não ter muita gente em volta. Porque senão fica todo mundo escutando, ficam querendo dar pitaco, essas coisas..., então a gente conversa com a família. OP1

\section{I.b) Compartilhar a informação de acordo com 0 entendimento do sujeito}

A comunicação deve ser feita de forma clara e em uma linguagem acessível às crianças e aos cuidadores. Os oncologistas entrevistados disseram haver uma conversa com os cuidadores, na qual relatam algumas coisas relevantes na doença e no tratamento, e outra conversa direcionada às crianças em uma linguagem que ela é capaz de entender.

A gente tem uma linguagem para o adulto e uma para a criança. OP4

Com os adultos é falado um pouco da doença. Falo do planejamento de exames, do tratamento, mas sem aprofundar muito nisso num primeiro momento. Se der muita informação de uma vez ele sai daqui sem entender nada. OP3

A gente explica pra criança numa linguagem que ela entenda. Por exemplo, pra falar da Leucemia eu falo de um jardim com rosas e que nesse jardim cresceu ervas daninhas.OP2

A última fala utiliza uma metáfora. A metáfora é a forma pela qual a criança se expressa, interpreta e comunica sua experiência de aflição. Desempenha um papel de ponte entre a singularidade da experiência e a objetividade da linguagem, permitindo organizar experiências subjetivas, de modo a transmiti-la aos outros - familiares, amigos, terapeutas ${ }^{13}$.

\section{I.c) Exploração do desejo de receber informações}

Após receber o diagnóstico, algumas perguntas frequentes são realizadas pelos cuidadores e pelas crianças. De acordo com os oncologistas pediátricos entrevistados, as perguntas mais frequentes das mães eram em relação à causa e à cura da doença.

Pergunta de quem é a culpa e por que foi que aconteceu. Sempre tentam achar um culpado. OP1

Alguns, mas não são todos que têm coragem, perguntam quais são as chances da criança morrer ou sobreviver. OP2
Se tem cura. É a primeira coisa que eles perguntam. OP5

Dependendo da idade, a preocupação das crianças é em relação ao agora. A maioria pergunta se vai poder continuar indo à escola ou se o tratamento vai causar algum desconforto como a dor. A queda de cabelo também parece ser preocupante para as meninas e adolescentes.

Se vai doer ou não. Eles querem saber mais a respeito do conforto ou se vai ter algum tipo de sofrimento. Muitos perguntam a questão do cabelo, principalmente as meninas. Acho que por questão de vaidade mesmo. OP3

As crianças perguntam muito pouco. Os adolescentes perguntam sobre o cabelo e se vão poder ir para a escola. Os pequenos perguntam se vai machucar. OP4

As perguntas realizadas pelas crianças e cuidadores são como pistas que oferecem ao médico as informaçōes sobre o que é importante abordar e o grau de aprofundamento que deve conter as informações.

\section{I.d) Acolher os sentimentos do sujeito}

Acolher os sentimentos dos pacientes e cuidadores é a última instância do processo da comunicação da verdade $^{12}$, porém esta questão não foi diretamente abordada por nenhum dos oncologistas pediátricos durante a entrevista.

Ao considerar a repercussão que a doença traz para a criança e sua família, o médico pode compreender melhor a aflição da família, a ansiedade dos pais e reconhecer os mecanismos de defesa que estão mobilizados e, assim, lançar mão de palavras mais acolhedoras. Parte da tarefa do médico de crianças é apoiar e encorajar a mãe, oferecer estímulos para que ela possa enfrentar a doença ${ }^{3}$.

\section{AS DIFICULDADES ENCONTRADAS NA ORGANIZAÇÃO E ESTRUTURA DO SERVIÇO DE ONCOLOGIA}

De acordo com os médicos entrevistados, são muitos os problemas encontrados para realizar o diagnóstico e o tratamento do câncer infantil. As queixas estão relacionadas às falhas na estrutura do SUS, ao diagnóstico tardio, às dificuldades de se pedir exames pelo SUS e à falta de medicação e desorganização do serviço.

Diagnosticar o câncer infantil não é fácil. Os sintomas gerais se confundem com os de outras doenças normais na infância, como febre, perda de peso, íngua, dor nas pernas e manchas roxas no corpo. O pediatra 
geral será, provavelmente, o primeiro médico procurado pela família, mas em toda a sua carreira deverá presenciar poucos casos de neoplasia maligna em relação às doenças comuns da infância, fazendo com que o câncer não seja a primeira hipótese considerada diante de queixas inespecíficas, porém, geralmente, quanto maior é o atraso do diagnóstico, mais avançada fica a doença, menores são as chances de cura e maiores serão as sequelas decorrentes de um tratamento mais agressivo.

Uma coisa que falta é o diagnóstico precoce, porque quem pega esses meninos que começam a aparecer no posto uma, duas e três vezes com a mesma queixa é o pediatra geral ou o médico da família, né. Então talvez um curso para esse pessoal, uma atualização... porque não é fácil fazer o diagnóstico de câncer, é difícil porque a maior parte dos sintomas da criança com câncer é sintoma habitual, tipo dor de cabeça e febre. Só que é aquele menino que volta cinco vezes no posto com dor de cabeça. OP1

A Constituição de 1988 estabeleceu que o direito à saúde fosse conquista universal, sem exclusōes. Essa determinação constitucional, que permitiria a efetiva implantação do Sistema Único de Saúde (SUS), foi construída a partir de duas garantias básicas: universalidade e descentralização. O exercício da equidade, apesar de sua importância, foi superado num primeiro momento pelas exigências da efetiva implementação da universalidade no sistema ${ }^{14}$, porém a implantação do SUS enfrenta dois descompassos gerenciais que sinalizam algumas incompatibilidades que, se não inviabilizam, ameaçam a estratégia de eficiência do programa; as pressōes por uma reforma do Estado que diminua custos como princípio norteador e a difícil convivência do princípio da descentralização com a crescente implementação da ideia de rede, aqui entendida não apenas como ligação entre esferas diferenciadas de gestão, mas como o modo pelo qual o acesso a um direito é exercido em rede "desde o planejamento, oferta e realização desse acesso". O princípio de "rede", essência do SUS, precisa ser complementar e não contraposto ao de descentralização, como algumas vezes tem ocorrido ${ }^{15}$.

Apesar do SUS falar que é descentralizado, eles centralizaram tudo. Então tudo que você vai fazer tem que pedir autorização para uma porção de comissão, e as respostas demoram um tempão comprometendo o tratamento e o prognóstico. As coisas poderiam ser autorizadas na própria instituição. O que o SUS faz é tirar a chance de cura de uma criança. Tem hora que a impressão que dá é essa mesmo. OP2

A gente vê algumas dificuldades em trabalhar com o câncer infantil. Primeiro que o paciente chega tarde pra gente. Existe o problema de exames que muitas cidades têm um número de cota por ano e que se der azar de mais crianças precisarem daquele exame, elas ficam sem fazer. E essas coisas fazem diferença no prognóstico da criança. Deveriam ter mais diálogos com os oncologistas pediátricos para saber das necessidades e das urgências do serviço. OP4

A dificuldade começa na admissão do hospital que a gente encontra dificuldade, pela estrutura mesmo do SUS. Depois tem a dificuldade de pedir exames. É tudo uma burocracia danada. Ainda faltam drogas para fazer a quimio. OP5

\section{HUMANIZAÇÃo}

A proposta da Política Nacional de Humanização (PNH) coincide com os próprios princípios do SUS, enfatizando a necessidade de assegurar atenção integral à população e estratégias de ampliar a condição de direitos e de cidadania das pessoas. Avançando na perspectiva da transdisciplinaridade, propóe uma atuação que leve à "ampliação da garantia de direitos e ao aprimoramento da vida em sociedade"16.

Todos os médicos se posicionaram quanto à situação de seus serviços frente à $\mathrm{PNH}$ :

A gente ainda está muito longe de humanizar o serviço. Precisa de alojamento por causa das condições que as mães e as crianças ficam aqui por até quatro meses sem ir em casa, vivendo numa poltrona. Paciente que trata fora de domicílio precisa de uma casa de apoio com uma estrutura legal, embora a gente tenha algumas poucas instituições associadas que têm casa de apoio que ajudam muito a gente.OP5

As casas de apoio recebem as crianças com câncer e seus cuidadores vindos de outras cidades para tratamento e estão enquadradas dentro das perspectivas do humanizaSUS. Auxiliam no tratamento, oferecendo acomodaçóes para pacientes que não necessitam de internação, proporcionando a liberação de leitos hospitalares que ficam disponíveis para crianças que realmente precisam ficar internadas. Também influenciam na melhoria das condições psicológicas e sociais das crianças que recebem o suporte para a saúde em um ambiente mais tranquilo e familiar do que o hospital. Como exemplos de casas de apoio que acolhem 
as crianças em tratamento de câncer em Belo Horizonte estão a Associação Unificada de Recuperação e Apoio (Casa AURA) e a Casa Beatriz Ferraz que contam com uma equipe de profissionais de saúde, incluindo psicólogos, terapeuta ocupacional, fisioterapeuta, enfermeiros, assistente social e outros.

A importância de uma casa onde as crianças com câncer possam morar durante o período de tratamento pode ser compreendida a partir da seguinte citação: " $A$ casa integra os pensamentos, as lembranças e os sonhos do homem. Sem ela, o homem seria um ser disperso. Ela é o corpo e alma. É o primeiro mundo do ser humano ${ }^{117}$. Um espaço acolhedor e familiar que, ao mesmo tempo, ofereça estrutura para o tratamento e desenvolvimento da criança pode tornar mais suportável esse momento por ela vivido. O local de moradia pode proporcionar conforto, descanso, saúde, saciedade, prazer, convívio etc., mas pode trazer também o contrário dessas sensaçōes, ou seja, desconforto, tristeza, desgaste, enfermidades etc. "Precisamos estar bem, precisamos ser bem acolhidos em nossa morada, a fim de que tenhamos possibilidade de desenvolver melhor nossas atividades, cuidar da nossa saúde e assim, quem sabe, modificar o mundo"18.

O humanizaSUS preconiza a formação de equipe multiprofissional de atenção à saúde para seguimento dos pacientes. Para os oncologistas pediátricos, essas equipes são de extrema importância, mas nem sempre se pode contar com elas.

É uma norma que o Ministério da Saúde coloca que muitas vezes não é respeitada aqui em Belo Horizonte. Que esses pacientes têm que ser tratados em centros especializados que têm uma estrutura própria para isso [...] um ambiente que esteja preparado para o tratamento com equipe multidisciplinar: pediatra, enfermeiro psicólogo, fisio, T.O, assistente social... se a criança e a família se encontram num ambiente assim, ela sabe que vai ter o atendimento adequado. $\mathrm{OP} 3$

Equipe multiprofissional, nossa, que luta que é ter uma equipe multiprofissional. $\mathrm{E}$ isso faz toda a diferença. Ter um oncologista, neuro, cirurgiāo, fisio, dentista, psicólogo, T.O, fono, nutrição. Se você tiver uma equipe multiprofissional bacana, tudo fica absolutamente melhor. OP1

Para os profissionais entrevistados, a organização e qualidade do serviço de oncologia pediátrico também deveriam fazer parte da Política Nacional de Humanização.
Mas acho que humanização não é só o bem-estar. É também dar a chance do paciente ser tratado corretamente, tipo não faltar drogas, não ter dificuldade de fazer o exame quando ele precisa ser feito. Isso também é humanizar.OP5

\section{CONSIDERAÇÕES FINAIS}

A comunicação do diagnóstico é um momento delicado para o paciente, familiares e médicos. $\mathrm{O}$ oncologista, pelo seu papel de retentor de conhecimento, deve oferecer aos pais a compreensão necessária sobre a doença para que estes possam suportar a doença e o tratamento do filho sem gerar sofrimento neles próprios.

O médico precisa partilhar a verdade do diagnóstico e prognóstico, por mais delicada e penosa que essa tarefa possa ser. A dificuldade dessa tarefa reside não somente na formulação verbal do diagnóstico e prognóstico ruim do ponto de vista técnico, mas, sobretudo, na carga afetiva de intenso sofrimento e desespero que sobre ele será imediatamente depositada após esta revelação. Deve dirigir esta família para a realidade e, ao mesmo tempo, respeitar que mantenham a esperança ${ }^{3}$.

De acordo com Hipócrates, o médico, diante da enfermidade, deve ter em mente dois objetivos: fazer o bem e não causar prejuízo. Para isso, é necessário oferecer ao médico e à equipe de saúde boas condiçôes de trabalho, liberdade para realizar os exames e intervençôes necessárias no momento que estes julgarem apropriado. O SUS deve servir à sociedade e oferecer a ela o que ela precisa no momento em que ela precisa. Necessita ser um cúmplice na promoção da saúde, prevenção e tratamento de doenças.

\section{REFERÊNCIAS}

1. Yantzi N, Rosenberg M, Burke S, Harrison M. The impacts of distance to hospital on families with a child with a chronic condition. Soc Sci Med. 2001;52(12):777- 91.

2. Caprara A, Rodrigues J. A relação assimétrica médicopaciente: repensando o vínculo terapêutico. Ciênc. Saúde Coletiva. 2004;9(1)139-46.

3. Leite AJM, Caprara A, Coelho Filho JM, organizadores. Habilidades de comunicação com pacientes e famílias. São Paulo: Sarvier; 2007.

4. Malta JDS, Schall VT, Modena CM. Câncer pediátrico: um olhar da família/cuidadores. Pediatr Mod. 2008;44(3):114-8.

5. Helman CG. Cultura, saúde e doença. Porto Alegre: Artmed; 2003.

6. Minayo MCS. O desafio do conhecimento: pesquisa qualitativa em saúde. São Paulo: Hucitec, Rio de Janeiro: ABRASCO; 2000. 
7. Triviños ANS. Introdução à pesquisa em ciências sociais: a pesquisa qualitativa em educação. São Paulo: Atlas; 1987.

8. Barona JL. Cancer patients and medical practice: some historical and cultural considerations. Ann N Y Acad Sci. 1997;809:17-29.

9. Suchman AL, Matthews DA. What makes the patientdoctor relationship therapeutic? Exploring the connectional dimension of medical care. Ann Intern Med. 1998; 108(1):125-30. Erratum in: Ann Intern Med 19881;109(2):173.

10. Instone SL. Perceptions of children with HIV infection when not told for so long: implications for diagnosis disclosure. J Pediatr Health Care. 2000;14(5):235-43.

11. Lecussan P. A criança e o diagnóstico: o pediatra deve contar à criança doente seu diagnóstico? Rev Assoc Med Bras. 2001;47(4):282-3.

12. Luz Bascunann RM. Comunicación de la verdad en medicina: contribuciones desde una perspectiva psicológica. Rev méd Chile. 2005;133(6):693-8.
13. Rabelo MCM, Alves PC, Souza IM. Experiência de doença e narrativa. Rio de Janeiro: Fundação Oswaldo Cruz; 2003.

14. Trevisan L. Das pressões às ousadias: o confronto entre a descentralização tutelada e a gestão em rede no SUS. Rev Adm Pública. 2007;41(2):237-54.

15. McGuire M. Managing networks: propositions on what managers do and why they do it. Public Adm Rev. 2002:62(2);135-43.

16. Ministério da Saúde, Secretaria de Atenção à Saúde. Política Nacional de Humanização. HumanizaSUS. Brasília (DF): Ministério da Saúde; 2006.

17. Bachelard G. A poética do espaço. São Paulo: Abril Cultural; 1978. [Coleção Pensadores].

18. Feltmann CS. Um olhar para o homem e sua morada [dissertação]. Rio de Janeiro (RJ): Fundação Oswaldo Cruz, Escola Nacional de Saúde Pública; 2002.

\section{Abstract}

The perception and reaction of a child towards the disease are influenced by the attitude of parents which, in turn, is determined by the doctor's attitude towards the disease and treatment. The objective of this article is to describe the time of notification of the diagnosis of childhood cancer to the patient and family and the main difficulties faced by pediatric oncologists in the treatment of children with cancer. As a criterion for choosing individuals for the interviews, the oncologists had to have specialization or residency in Pediatric Oncology. 5 oncologists were interviewed. The interviews were semi-structured and recorded with the permission of the interviewees and the analysis of the material was made by examining issues. This analysis allowed the identification of three broad categories in relation to time to confirm the diagnosis of childhood cancer and the difficulties encountered in the treatment of the disease: 1 ) The truth must be said to both children and parents; 2) The difficulties encountered in the organization and structure of the department of oncology; 3) The issue of humanization. The communication of the diagnosis is a delicate instance for the patient, family and doctors. The oncologist, for his role as keeper of knowledge, must offer parents the necessary understanding about the disease so that they can handle the disease and treatment of the child without suffering they generate themselves. Regarding the difficulties encountered by doctors in the treatment of cancer, the SUS have to be an accomplice in the promotion of health, prevention and treatment of diseases.

Key words: Medical oncology; Diagnosis; Pediatrics; Cost of Illness; Child care; Neoplasm 